\title{
Geiger-mode APD as a RICH Photodetector
}

T. Iijima ${ }^{a *}$, R. Dolenec ${ }^{b}$, K. Hara ${ }^{a}$, S. Korpar ${ }^{b, c}$, P. Križan $^{b, d}$, Y. Mazuka ${ }^{a}$, R. Pestotnik ${ }^{b}$, A. Petelin ${ }^{b}$, M. Yamaoka ${ }^{a}$

${ }^{a}$ Department of Physics, Nagoya University, Nagoya, Japan

${ }^{b}$ Jožef Stefan Institute, Ljubljana, Slovenia

${ }^{c}$ Faculty of Chemistry and Chemical Engineering, University of Maribor, Maribor, Slovenia

${ }^{d}$ Faculty of Mathematics and Physics, Ljubljana, Slovenia

E-mail: iijima@hepl.phys.nagoya-u.ac.jp

Recently developed APDs operated in the limited Geiger mode have been tested in view of measuring Cherenkov photons in a Ring Imaging Cherenkov (RICH) detector with aerogel radiator. Our studies show that the Geiger-mode APDs are a possible photodetector candidate for RICH counters with aerogel, and significantly improve the RICH performance.

International workshop on new photon-detectors PD07

June 27-29 2007

Kobe University, Kobe, Japan

* Speaker.

Corresponding author. 


\section{Introduction}

Recently developed APDs operated in the limited Geiger mode, referred to as "G-APD" hereafter, have high gain $\left(\sim 10^{6}\right)$, high photodetection efficiency $(\sim 40 \%$ at peak), and good time resolution $(\sim 100 \mathrm{ps})$. Moreover, they are insensitive to magnetic fields, which is a great advantage over other position sensitive photodetectors. These features attract great interest in application of G-APDs to detect a single photon in Ring Imaging Cherenkov (RICH) detectors. The major concerns are the rather small size $\left(\sim 1 \times 1 \mathrm{~mm}^{2}\right)$ and a very high dark rate $\left(\sim 10^{6} \mathrm{~Hz} / \mathrm{mm}^{2}\right)$.

In this paper, we discuss possibility of using G-APDs in the case of a proximity focusing RICH with aerogel radiator. Such a detector has been proposed for future upgrade of the Belle detector at the KEK B-factory [1]. In the development of this detector, we introduced a novel idea of using multiple aerogel layers of varying refractive index $(n)$, to increase the number of detected photons $\left(N_{p e}\right)$ without deteriorating the Cherenkov angle resolution per photon $\left(\sigma_{\theta}\right)$ due to the emission point uncertainty [2]. In a beam test using an array of multi-anode PMTs (Hamamatsu H8500) as the photodetector, we obtained $N_{p e}=9.6$ and $\sigma_{\theta}=14.4 \mathrm{mrad}$ for a combination of $n=1.047 / 1.057$ aerogel layers, each being $2 \mathrm{~cm}$ thick. More recently, we demonstrated that Cherenkov photons produced in the entrance window of the PMT provide an excellent time-of-flight measurement [3].

Figure 1 shows the concept of a RICH photodetector based on an array of G-APDs under investigation. A light collector, either a trapezoid light guide or a lens, is attached to each G-APD channel, to enlarge the geometrical acceptance, and hence to increase the number of signal hits per track. We consider using a narrow time window $(\lesssim 10 \mathrm{~ns})$ to reduce accidental overlaps of the dark noise to an acceptable level. The light collector serves also as a light emitter for the time-of-flight measurement, as mentioned above.

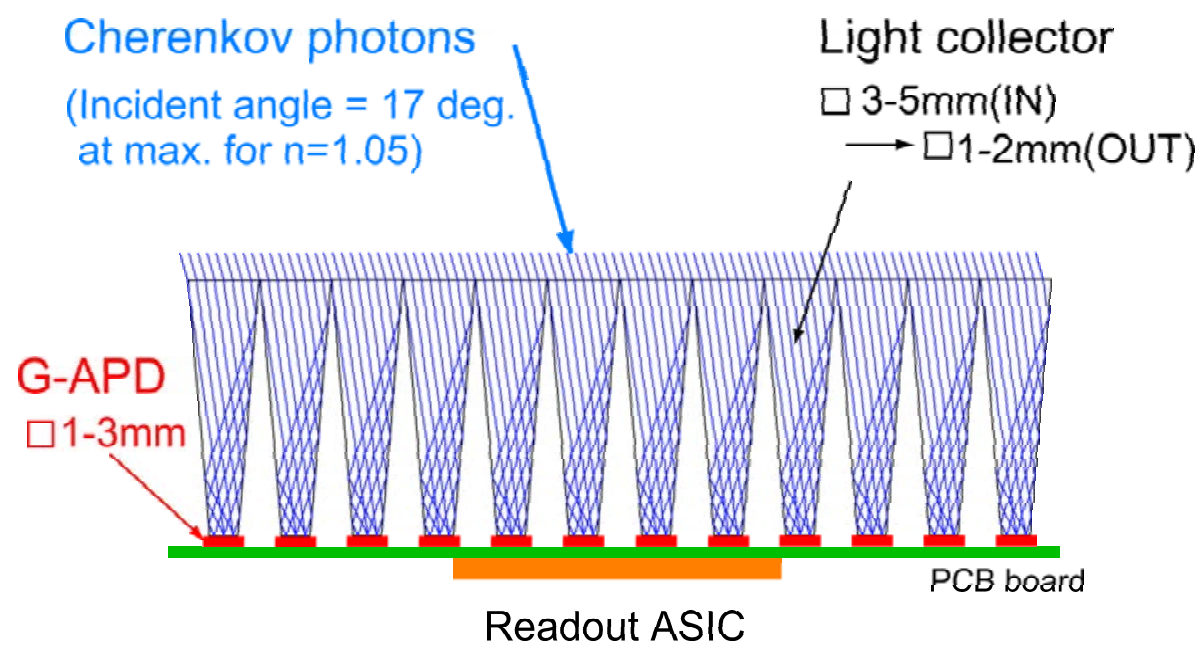

Figure 1: concept of a RICH photodetector based on a G-APD array.

\section{G-APD characterization}

In order to clarify the feasibility of using G-APDs for RICH counters, we have measured 
basic properties of several G-APD samples; MPPC from Hamamatsu Photonics with 100 and 400 pixels, MRS-APD from CPTA Moscow with 556 pixels, and SiPM from MePhI/PULSAR with 1156 pixels.

\subsection{Photodetection efficiency}

The photodetection efficiency (PDE) is defined as $\mathrm{PDE}=\mathrm{QE} \times \varepsilon_{\text {geom }} \times \varepsilon_{\text {Geiger }}$, where $\mathrm{QE}$ is the quantum efficiency, $\varepsilon_{\text {geom }}$ is the geometrical efficiency, and $\varepsilon_{\text {Geiger }}$ is the probability of inducing a Geiger discharge when an electron-hole pair is generated. Figure 2-a) shows the measured PDE for a MPPC sample (400 pixel). The PDE is estimated by comparing the output currents from a G-APD sample and the reference PIN diode when both are illuminated with the light from a photospectrometer. The measured PDE reaches $45 \%$ at the peak $(\sim 460 \mathrm{~nm})$, and remains higher than $20 \%$ for the wavelength region from $320 \mathrm{~nm}$ to $660 \mathrm{~nm}$. The high sensitivity over a wide wavelength region results in a considerably increased value of $N_{p e}$ for a $\mathrm{RICH}$ with aerogel radiator 1 .

\subsection{Gain and noise rate}

Figure 2-b) shows correlation between the gain and the noise rate (with a threshold below 1 p.e.) for the MPPC samples. For both 100- and 400-pixel samples, recent products have much improved noise rate, about 5-6 times lower than the old samples. For instance, the noise rate for a 100 -pixel sample is $200 \mathrm{kHz}$ when operated at the gain of $10^{6}$. The significant improvement in the noise rate is very encouraging for the application of G-APD to RICH detectors.
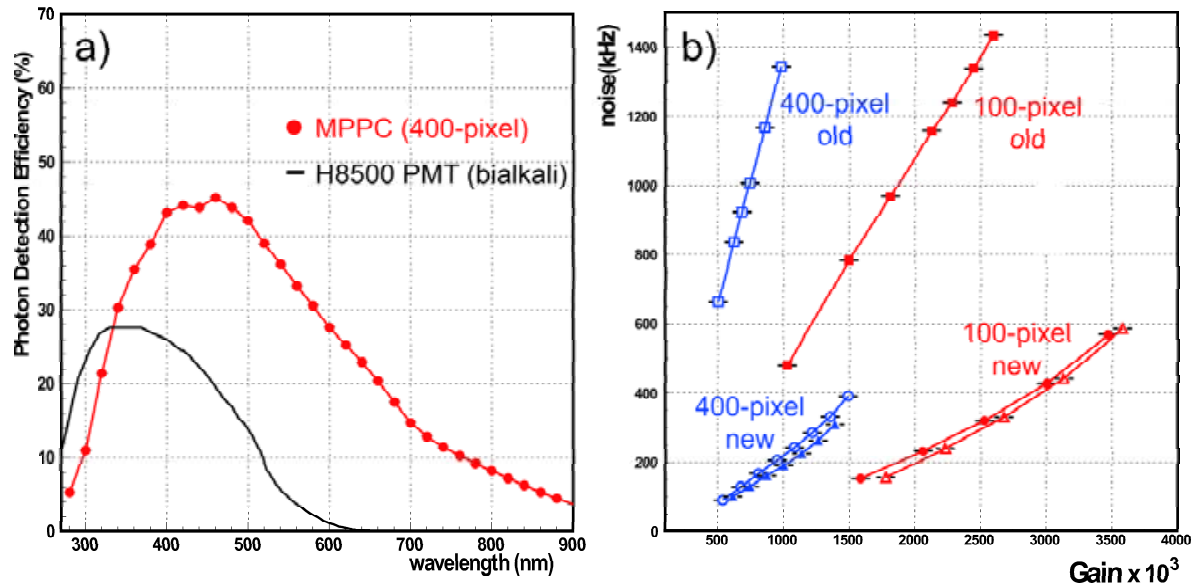

Figure 2: a)The measured photodetection efficiency (PDE) and b) correlation between the gain and the noise rate for the MPPC samples.

\footnotetext{
${ }^{1}$ The PDE based on the current measurement includes contribution from after pulses and cross-talk among micro pixels. Corrections due to such effects are under investigation.
} 


\subsection{Time resolution}

Figure 3 shows the time distribution for a single photon irradiation by a pulse laser at $636 \mathrm{~nm}$ and $410 \mathrm{~nm}$. The result is shown for a MPPC sample, and is corrected for the time walk. The obtained time resolution is about $110 \mathrm{ps}(\sigma)$. Therefore, G-APD is useful also for TOF measurement.
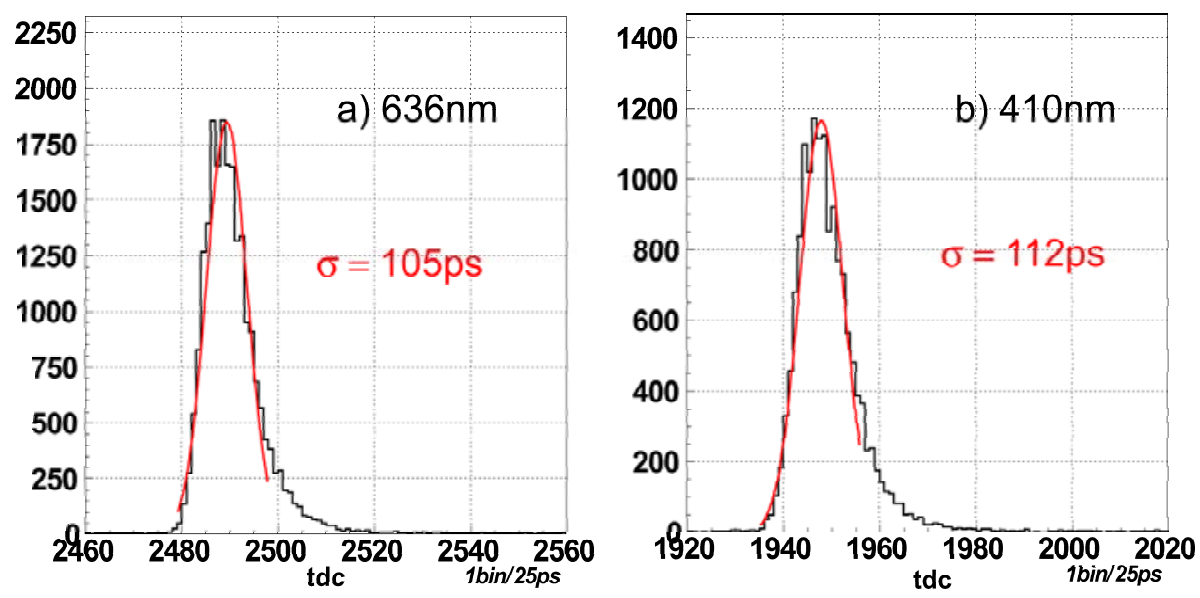

Figure 3: Time distribution for a single photon irradiation by a pulse lalser at 636 and $410 \mathrm{~nm}$ for a MPPC sample.

\subsection{Surface scan}

Figure 4 shows the position dependence of the single-photon response for different types of G-APDs. The variation of sensitivity reflects the underlying structure of the device. For most of the devices, we observe the position response is fairly constant for pixels, although there is somewhat more variation within each pixel.

\section{Light collection system}

We are also designing the light collection system to enlarge the geometrical acceptance and improve signal to noise ratio. For instance, Figure 5 shows results of a ray-trace simulation for trapezoid light guides with a $5 \mathrm{~mm} \times 5 \mathrm{~mm}$ input and $2 \mathrm{~mm} \times 2 \mathrm{~mm}$ output surfaces, and with and without a lens on the input surface. The incident angle of the incoming ray is 0.3 radian, which is close to the maximum incident angle of a Cherenkov light in the RICH detector designed for the Super-KEKB. The figure shows that about $90 \%$ light collection efficiency is achievable.

\section{Expected RICH performance}

The RICH performance with the array of G-APDs and the light guides, as presented in Figure 1, has been estimated based on a Monte Carlo (MC) simulation. The simulation includes the measured properties of the MPPC sample, and light transportation inside the trapezoid light guide, as described in the previous sections. Figure 6-a) shows the wavelength distribution of photons, 

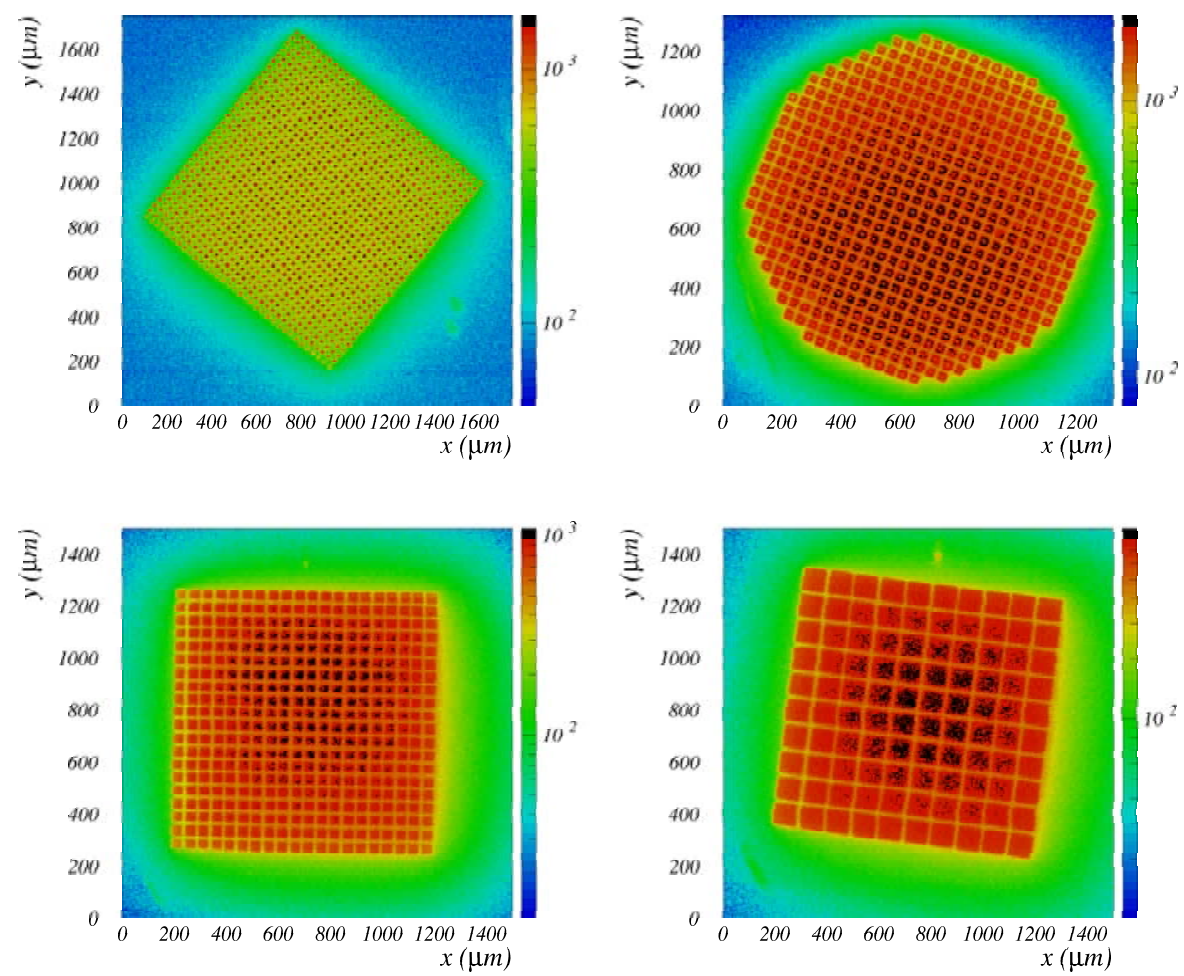

Figure 4: Surface sensitivity to single photons for different G-APDs; SiPM from MEPhi/PULSAR (topleft), MRS-APD from CPTA Moscow (top-right), 400-pixel MPPC (bottom-left), and 100-pixel MPPC (bottom-right) from Hamamatsu Photonics.
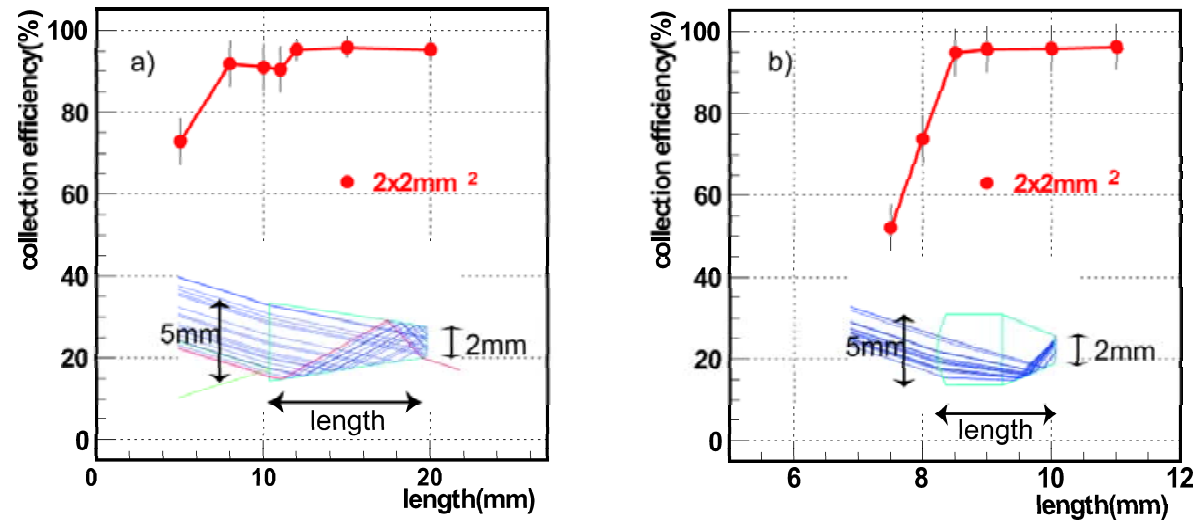

Figure 5: Transmission estimated by a ray-trace simulation for trapezoid light guides a) without and b) with lens on the input surface. 
reaching to the photodetector, and detected by the array of light-guides $\left(5 \times 5 \rightarrow 2 \times 2 \mathrm{~mm}^{2}\right)$ and MPPCs. The same distribution in the case of the multi-anode PMT array is also shown for comparison. The figure shows that the proposed photodetector gives about 4 times larger light yield than the multi-anode PMT, because of the high photodtection efficiency, as presented in Figure 2-a).

Effects of the drak noise have been studied in a separate MC simulation, in which random background hits are embeded. Figure 6-b) shows the kaon identification efficiency at $1 \%$ pion misidentification probability as a function of particle momentum for different background hit occupancy. In the simulation, $N_{p e}$ per ring is assumed to be 20 conservatively. For the recent MPPC sample with 100 pixels, the measured noise rate is about $200 \mathrm{kHz} / \mathrm{mm}^{2}$ at $10^{6}$ gain, leading to 0.8 $3.2 \%$ occupancy for $2 \times 2-4 \times 4 \mathrm{~mm}^{2}$ G-APD pad size for a $10 \mathrm{~ns}$ time window. Although some loss in the efficiency is seen in the high $(\gtrsim 4 \mathrm{GeV} / c)$ and the low $(\lesssim 1.5 \mathrm{GeV} / c)$ momentum regions, the efficiency close to $100 \%$ is forseen in the momentum region from 1.5 to $4 \mathrm{GeV} / c$. A tighter time window, either in electronics or in the offline analysis, will help to minimize the efficiency loss. Further reduction in the dark noise rate in the production is of course helpful. The usage of the TOF information will improve significantly the performance in the low momentum region.
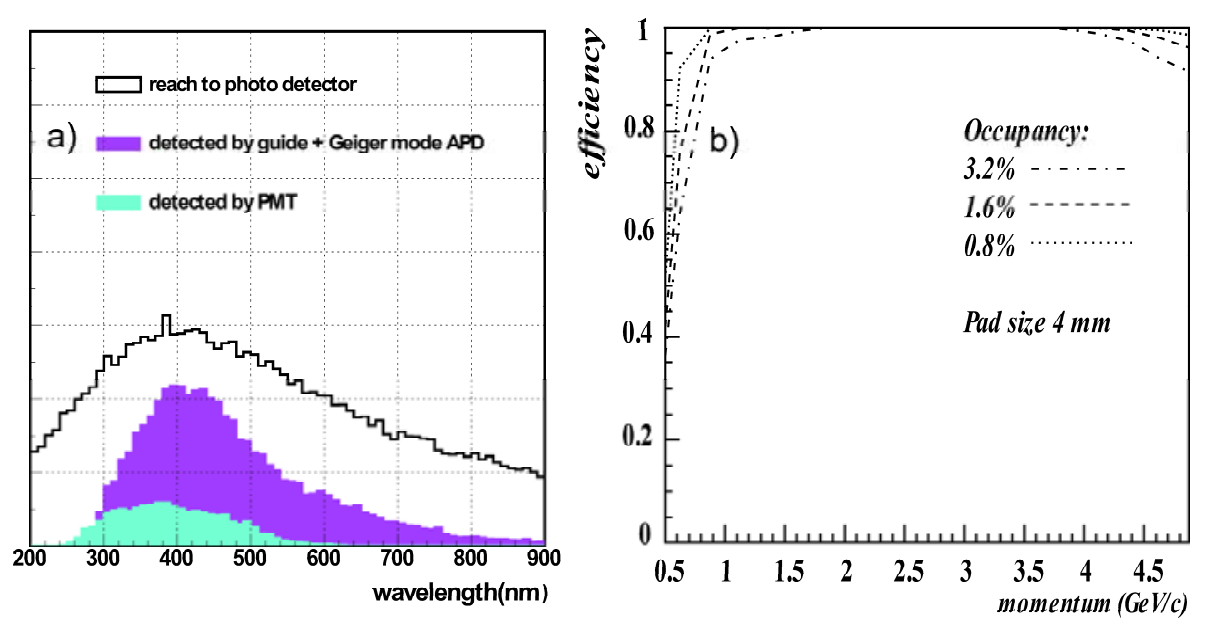

Figure 6: a) Wavelength distribution of photons, reaching to the photodetector, detected by the array of the trapezoid lightguides and MPPC. The same distribution in the case of the muti-anode PMT array is also shown for comparison. b) kaon identification efficiency at $1 \%$ pion misidentification probability as a function of particle momentum for different levels of background hit occupancy.

\section{Summary}

We have studied the G-APDs for application to RICH counters, in particular, the proximity focusing RICH with aerogel radiator. We consider using an array of G-APDs and light-guides to measure the position of a single photon. In addition to the insensitivity to the magnetic field, GAPD has great benefits; the high PDE in a wide wavelength region will give significant increase in $N_{p e}$ (about 4 times more compared to a conventional PMT), and the good time resolution of about $110 \mathrm{ps}(\sigma)$ enables us to measure TOF. The dark noise rate has been significantly improved for the 
recent MPPC samples from Hamamatsu (about $200 \mathrm{KHz} / \mathrm{mm}^{2}$ at $10^{6}$ gain), and in a tolerable range if a narrow time window of $10 \mathrm{~ns}$ or less is used. A ray-trace simulation indicates that a trapezoid lightguide, optionally with a lens, has good light transmission for the range of incoming rays in the actual application $(\sim 90 \%)$. If G-APDs with larger size become available in the near future, it will make the design of the RICH photodetector more flexible, and make construction of a large array more realistic.

The authors would like to thank the members of the high energy physics group (N-lab.) Nagoya university, the Belle ACC group, the KEKDTP RD-photon group for their cooperation to this R\&D. They also thank Hamamatsu Photonics and Yuri Kudenko for providing G-APD samples. This work is supported in part by a Grant-in-Aid for Scientific Research on Priority Areas "New Developments of Flavor Physics" from the ministry of Education, Culture, Sports, Science and Technology of Japan.

\section{References}

[1] K. Abe et al., Letter of Intent for KEK Super B Factory, KEK report 2004-04, http://belle.kek.jp/superb/.

[2] T. Iijima, S. Korpar et al., Nucl. Instr. and Meth. A548 (2005) 348.

[3] S. Korpar et al., Nucl. Instr. and Meth. A572 (2007) 432. 